\title{
DEVELOPMENT OF A ROUTE CROSSING TOOL FOR SHARED AIRSPACE ENVIRONMENTS
}

\author{
Daphne Rein-Weston, San Jose State University/NASA Ames Research Center, Moffett Field, CA \\ Richard Jacoby, ASRC Research and Technology Solutions, Moffett Field, CA \\ Eric Chevalley, Albert Globus, Hyo-Sang Yoo, Bonny Parke, Paul Lee, Faisal Omar, Joshua M. Kraut, \\ Nancy Bienert, Abhay Borade, Conrad Gabriel, \& Kari Gonter, San Jose State University / NASA \\ Ames Research Center, Moffett Field, CA \\ Everett Palmer, NASA Ames Research Center, Moffett Field, CA
}

\begin{abstract}
In current-day Terminal Radar Approach Control (TRACON) operations, departure and arrival controllers maintain separate and dedicated airspace for their respective traffic flows. Although this practice has obvious safety features, it also leads to inefficiencies; for example, departure aircraft may be routinely capped beneath arrival airspace. With the right decision-support and coordination tools, departures could continue to climb through arrival airspace when sufficient gaps exist. Previous studies of 'shared airspace' have examined pre-arranged coordination procedures, as well as tools that gave feedback to the controllers on where gaps between arrivals were located and whether the departure aircraft could be scheduled to fly through those gaps $[1,2,3,4]$. Since then, the Route Crossing Tool (RCT) has been developed to allow controllers to assess multiple pre-defined route options at points where the arrivals and departures cross, thereby increasing the possibility of climbing a departure through an arrival gap.

The RCT aids in ensuring lateral separation between departure and arrival aircraft that pass through the same altitude. Since the RCT can be applied tactically, it can enable aircraft to fly through arrival flows even if these aircraft depart outside scheduled times. The RCT makes use of a set of predefined parallel departure routes crossing the arrival flow at equidistant intersecting points on the arrival route. The RCT uses the Estimated Time of Arrival (ETA) of the departure aircraft at each intersecting point to calculate the lateral separation with the neighboring arrivals when it crosses that point; this information is graphically displayed to the controller. Additionally, the RCT incorporates forecast winds in its ETA predictions.
\end{abstract}

Multiple prototypes of the RCT have been iteratively developed with feedback from Subject Matter Experts (SMEs). This paper presents the final design, the design process, and lessons learned. Initial results from a simulation suggest that the tool was successful in helping controllers to safely climb more aircraft. Controller feedback on the tool was also positive.

\section{Introduction}

In today's TRACON operations, arrival and departure flows are managed by separate air traffic controllers, each with their own dedicated airspace. This segregation leads to suboptimal routes in metroplex airspace where multiple airports share limited resources, such as waypoints, paths or airspace [1]. Examples of these suboptimal routes are departure routes tunneling under arrival routes to maintain procedural altitude separation at common waypoints. While procedurally separating departure and arrival routes with altitudes is safe, departure aircraft could climb a more efficient route when no arrival traffic is present.

In an effort to improve efficiency, previous studies have examined the procedures and feasibility of desegregating these operations by optimizing trajectories while using temporal separation rather than spatial separation $[2,3,4,5,6]$. Other studies have modeled the costs and benefits of unrestricted climb profiles $[7,8,9]$.

Human-in-the-loop (HITL) simulations were conducted in 2012 and 2013 in the Airspace Operations Laboratory (AOL) at NASA Ames Research Center on the Multi-Aircraft Control System (MACS) platform to investigate temporally separated solutions in a simulated San Francisco Bay Area airspace $[2,3,4,10]$. The first two Sharing of 
Airspace Resources (SOAR) simulations manipulated departure times at a San Jose airport to allow departure aircraft to fly through gaps in arrival flows to San Francisco and Oakland airports. Controllers were given tools and procedures to help them decide whether to climb aircraft. The first SOAR study found that controllers climbed aircraft opportunistically. They vectored aircraft when possible and cleared aircraft early enough to climb aircraft above the arrival traffic. Controllers anticipated separation while at times losing positive control as they were not always able to keep vertical or lateral separation [2]. In the second SOAR study, controllers were restricted to stay on the routes, but were given decision support tools to help them decide whether and when to climb aircraft. Controllers were given 3 types of tools: 1) timelines, 2) tie-boxes and 3) a conflict-probe. The timelines showed both arrival and departure ETAs at the crossing waypoint. The tie-boxes were static tools that showed tie-points between arrivals and departures at a given time. The tie-boxes were drawn on the videomap displayed on the controller's scope. The conflict-probe was a dynamic tool that showed all aircraft predicted to get within a certain lateral and vertical distance of each other. Results showed that both the static and dynamic visual tools helped controllers to better assess separation. However, controllers tried to climb aircraft early so they could maintain separation by climbing departures above (not through) the arrival stream. Separation was lost in a few instances [3]. This was due to unpredictable climb performances as well as not having any accurate estimates of separation between departing and arriving aircraft.

The tie box was a passive decision-support tool. Assessment of aircraft separation was based on the fixed locations of aircraft and did not take into account wind or aircraft climb speeds. This led to uncertainties in prediction. The conflict-probe was an active tool that took into account predicted aircraft trajectories but it did not give path options to maintain separation.

These studies showed the need for a dynamic decision-support tool, one that used aircraft estimated times of arrival (ETAs) to assess aircraft separation, as well as offered trajectory and path options to controllers to enable them to select the best crossing point. Such a tool would also take into account separation standards and winds, and would thus ensure that controllers could keep positive control of an aircraft in an unrestricted climb.

Therefore a new decision-support tool, the Route Crossing Tool, was developed and used during the third SOAR simulation in June of 2014. This tool was based on predicted ETAs at the route crossing points, which enabled a more accurate assessment of where and when to cross a departure through an arrival stream. A simulated New York TRACON airspace was selected to test the operational use of the concept, since this airspace is known for its many complexities and challenges. While subsequent publications will examine the specific results of the 2014 HITL, this paper's focus is on the design iterations of the RCT. It should be noted that although the New York metroplex was used to inform the design of this tool, the functions and benefits of the tool should be generalizable to other airspaces with crossing arrival and departure streams.

\section{Route Crossing Tool Development}

The RCT is a ground-based decision support tool. Its primary function is (1) to assess ETAs of arrival and departure aircraft at route crossing points, and visually provide estimated separation values to controllers. The RCT also (2) assesses estimated separation for multiple crossing points and give controllers options on where to cross an arrival stream and still maintain lateral separation between aircraft. Both of these functions are tactical.

A strategic function is also provided by this tool. While the tool assesses the ETA of aircraft at multiple crossing points, it also (3) predicts the delay aircraft will incur by measuring the flying time of the multiple preset trajectories to a common waypoint, e.g., to a departure fix.

The RCT development began by addressing the fundamental problem of ensuring adequate separation between departures and arrivals through temporal rather than procedural altitude separation constraints at a crossing point in space. The crossing point in the simulation was where the departure aircraft from Newark Liberty International (EWR) fly under arrival aircraft to LaGuardia International Airport (LGA). It should be noted that in this concept, although the desired separation is temporal and lateral, the spatial/altitude separation remains available in case temporal separation is shown not to be a viable option. 
The RCT concept assumes that aircraft trajectories for both arrival and departures can be predicted. It assumes that multiple path options can be pre-defined in a shared airspace where both arrival and departure aircraft fly. Finally, it assumes that controllers can update Standard Instrument Departure (SID) routes on the ground system.

The main components of the RCT are the multiroute options, route geometry, route length, and the aircraft's trajectory and ETA prediction. After a discussion of the benefits of multi-route options, each will be discussed below, followed by a discussion of the graphical user interface.

\section{Multi-route Options}

\section{Benefits}

The multi-routes are an intrinsic part of the RCT: the various lateral paths combined with speed define the recoverable range of schedule discrepancies for which the route options can compensate. This is especially important considering the inherent uncertainty in departure take off times.

The need for a controller's flexibility in safely maneuvering a departure through an arrival flow at co-altitude depends largely on the density of the arrival flow. Available path options for the departure aircraft add to the controller's flexibility. Instead of having a single path option for the departure (and therefore one departure-arrival path intersection point), multiple paths are pre-defined so that the controllers may choose from various path options.

One can ask whether it is better to have these pre-defined paths or to have the controller vector the departure through the arrival flow. Vectoring would provide a significant benefit in that it would remove the pre-defined path constraint and therefore the defined window of time to cross the departure-arrival intersection point. However, pre-defined routes allow pilots to fly selected SIDs using their Flight Management System (FMS), rather than having to follow radar vectors. Additionally, a known "family" of routes from which the controllers can choose, simplifies the ground-to-ground and ground-to-plot coordination.

\section{Route Geometry and Separation Rules}

The geometric design of the path options has a significant bearing on the feasibility of the RCT concept. To guarantee that the closest point of approach of a departure-arrival aircraft pair will not be less than a certain distance (e.g., acceptable predicted separation values), the tool has to take into account the angles formed by the respective paths that the aircraft follow.

The closest point of approach between a pair of arrival and departure aircraft is relative to the angle between the two aircrafts' headings. The optimal angle is when paths are perpendicular.

The lateral distance between two aircraft can be defined as:

The horizontal separation in time, $S(t)$, between arrival and departure aircraft is defined as:

$$
\text { (1) } \mathrm{S}(t)=\sqrt{ }\left[x_{\mathrm{A}}(t)-x_{\mathrm{D}}(t)\right]^{2}+\left[y_{\mathrm{A}}(t)-y_{\mathrm{D}}(t)\right]^{2}
$$

In the equation above, $x(t)$ and $y(t)$ indicate the horizontal positions of departure aircraft $(D)$ and arrival aircraft $(A)$ throughout time $(t)$.

The reference frame is fixed at the initial position of aircraft where the initial separation between aircraft at $t=0$ is determined. The position of aircraft at any time $t$ after flying its own straight path is as follows:

$$
\begin{aligned}
& \text { (2) } x(t)=x(0)+v \cos (\theta) t \\
& \text { (3) } y(t)=y(0)+v \sin (\theta) t
\end{aligned}
$$

In the equations above, $v$ represents the speed of aircraft and the $\theta$ denotes the angle between due north and the direction of the aircraft (i.e., the current heading). Depending on the initial positions of the aircraft and relative angle between headings of the aircraft, the separation between aircraft on the route crossing path is continuously changing as they fly.

With the above equations, the distance between two aircraft flying on crossing paths can be computed. The closest point of approach is after one aircraft crosses in front of the other. The distance decreases until this point and then increases after this point. Figure 1 shows the progression of relative distance during 60 seconds between two aircraft flying at 250 knots on a 90 degree crossing path, for various distances of initial separation between aircraft. The initial position $(t=0)$ of the departure aircraft is at the crossing point and the initial position $(t=0)$ of the approaching arrival aircraft is at various 
distances from the crossing point $(3,4,5$, and 5.66 mile). The figure shows how the separation of the aircraft continuously evolves over time.

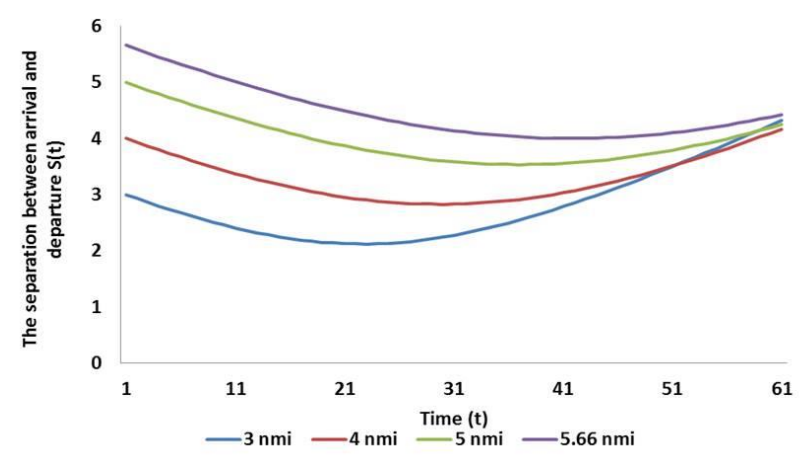

\section{Figure 1. Separation Between Aircraft Over Time}

The geometrical design of the path has a significant effect on the separation between the aircraft. Figure 2 shows the impact of the relative angle between aircraft paths $(60,70,80$, and 90 degrees). It can be seen that when the paths are perpendicular, the separation between aircraft is most assured since the smaller the angle, the smaller the closest point of approach (in time) and the earlier it occurs.

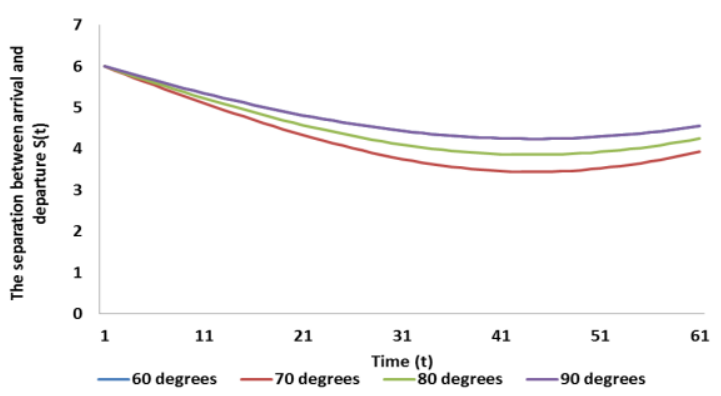

Figure 2. Angles Between Headings \& Separation

The distance of the closest point of approach is related to the separation standards that TRACON air traffic controllers follow. In TRACON airspace, the standard separation minima is $3 \mathrm{~nm}$ laterally and $1,000 \mathrm{ft}$ vertically. Controllers also can apply diverging separation rules to aircraft that are on crossing headings with an angular difference of at least 15 degrees. Aircraft on converging courses need to maintain standard separation, but as soon as one aircraft has crossed directly in front of the other, they are said to be diverging and the standard separation can be discontinued (See Section 5-5-7 in FAA Order 7110.65V [12]).

Figures 3 and 4 illustrate two cases where controllers used the divergence rule to climb a departing aircraft. In these examples, departing aircraft have an altitude clearance to $8,000 \mathrm{ft}$ and the arriving aircraft is at or above $9,000 \mathrm{ft}$ so that both aircraft are altitude separated until the point of divergence. In Figure 3, as soon as the arrival aircraft (AWI 428) crosses in front of the departing aircraft (UAL 615) at MOFT 4, the departing aircraft has 'course divergence' with the arrival and can be cleared to an altitude above $8,000 \mathrm{ft}$.

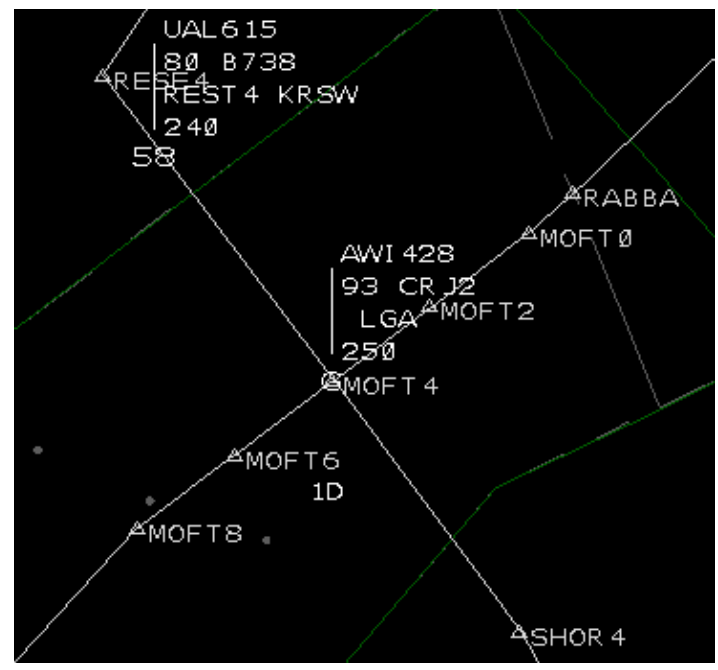

Figure 3. UAL615 Departure Behind Arrival

In Figure 4, as soon as the departing aircraft (CHQ374) crosses in front of the arriving aircraft (DAL486), it has 'course divergence' and can then be cleared to an altitude above $8,000 \mathrm{ft}$.

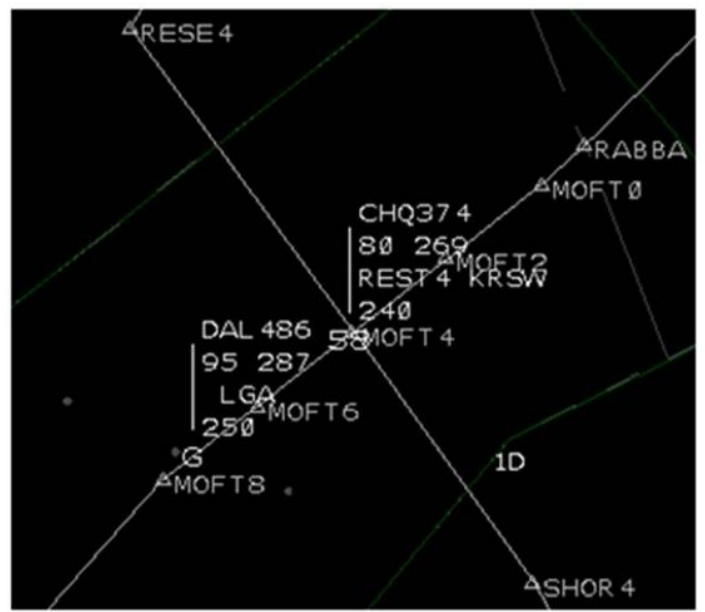




\section{Figure 4. CHQ374 Departure in Front of Arrival}

Figure 5 illustrates a case where the controller climbed the departure (CJC648) in front of an arrival (AWI481) before divergence was established. A conservative interpretation of the divergence rule is that in this case, the departure cannot violate standard separation minima with the arrival aircraft even after it crosses in front of the arrival. In this example, when CJC648 crossed MOFT4 it was $7.0 \mathrm{~nm}$ in front of AWI481. The closest point of approach of these two aircraft occurred about 50 seconds later when the two aircraft were $4.6 \mathrm{~nm}$ apart, so no separation minima were violated.

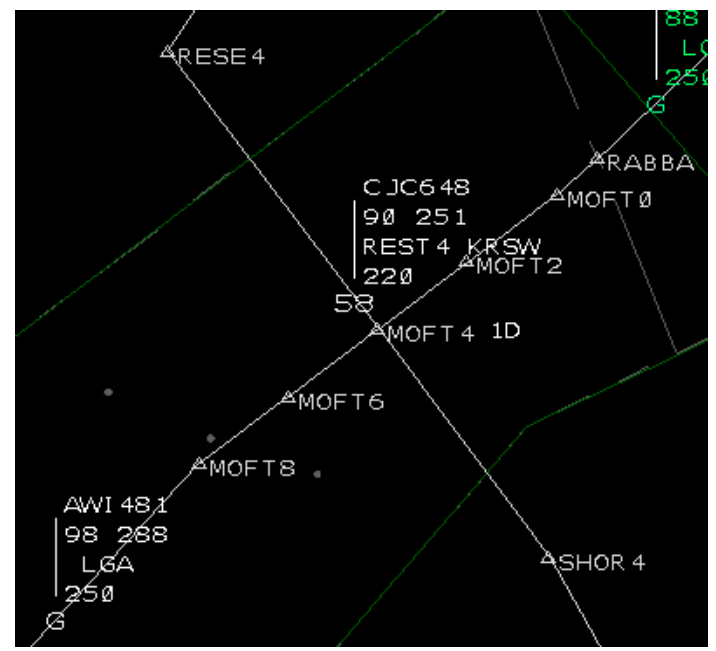

Figure 5. Departure in Front of Arrival Before Divergence

To illustrate further, Figure 6 shows two configurations of arrival (A) and departure (D) aircraft. The left side of the figure indicates the initial positions of the aircraft at the crossing time, $t_{c}$, and the right side of the figure represents the future minimum separation positions of the aircraft at time $t_{\min }$. The departure aircraft is red and the arrival is blue, with arrows indicating the headings. $S_{c}$ is the crossing point separation and $S_{\min }$ is the minimum separation.

In Figure $6 \mathrm{~A}$, the departure aircraft has climbed in front of the arrival aircraft before divergence was established. In this case, the separation at crossing, $S_{c}$, must be large enough so that the subsequent minimum separation, $S_{\min }$, is greater than $4 \mathrm{~nm}$. If the aircraft are traveling at equal speeds and at constant headings (with a course crossing angle of $\theta$ ), the required separation at crossing is
(4) $S_{c}=S_{\min } / \cos (\theta / 2)$.

In the case shown, where the course crossing angle is $90^{\circ}$, and the required $S_{\min }$ is 4 , the required $S_{c}$ is 5.6 nm.

In Figure $6 \mathrm{~B}$, the departure will pass behind the arrival. If the controller waits a few seconds until the routes have diverged to climb the departure, the required $S_{c}$ is 4 and the $S_{\min }$ is $2.8 \mathrm{~nm}$ where there is a $90^{\circ}$ angle.
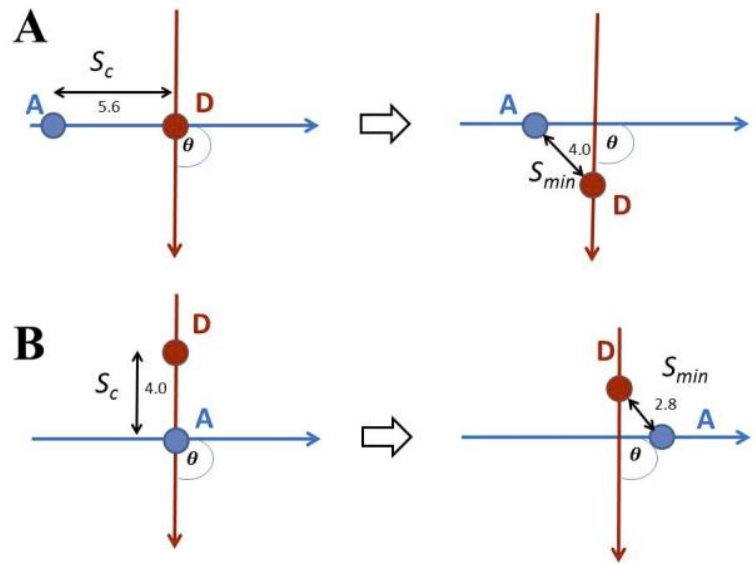

Figure 6. Route Crossing Separation Geometry

As can be seen, the RCT was designed to support TRACON separation rules and the types of decisions controllers make. It uses trajectory and ETA predictions to provide estimates of where the lead and trail arrival will be when the departure crosses the arrival flow.

\section{Route Length}

The departure aircraft's flying time is affected not only by the route geometry formed by the departure routes crossing the arrival flow, but also by the length of each departure route from the airport to the point at which it crosses the arrival route. While non-parallel routes can be considered, equivalent flying time differences over alternative routes within the departure route "family" contributes to the transparency and ease of use of the RCT. Figure 7 below is an illustration of a preliminary departure route family in the New York TRACON airspace. Controllers have a set of five parallel routes from which to assign aircraft departing from Newark Runway 22R. The departure aircraft fly southwest until turning left at the particular 'RESE' waypoint which corresponds to the SID issued by the controller. 
The parallel route structure allows for adjustments made to departures which can alter the tie point between them and an arriving aircraft. For example, a departure aircraft from EWR will intersect the LGA arrival flow approximately a minute sooner if the controller instructs a left turn at RESE0 rather than RESE4. (To get to RESE4, the departure has to fly an extra $4 \mathrm{~nm}$ at a speed of approximately $4 \mathrm{~nm}$ per minute.) Although the departure will get to MOFT0 about 1 minute earlier than it would get to MOFT4, the arrival will get to MOFT0 about 1 minute later than MOFT4. Hence the time between the aircraft on the timeline will increase by two minutes from MOFT0 to MOFT4. The relative change in time enabled by the various route crossing points can be used to correct for departure time errors. The set of routes as a whole, however, form a parallelogram which allows for the overall time to a downstream meter fix to remain unchanged.

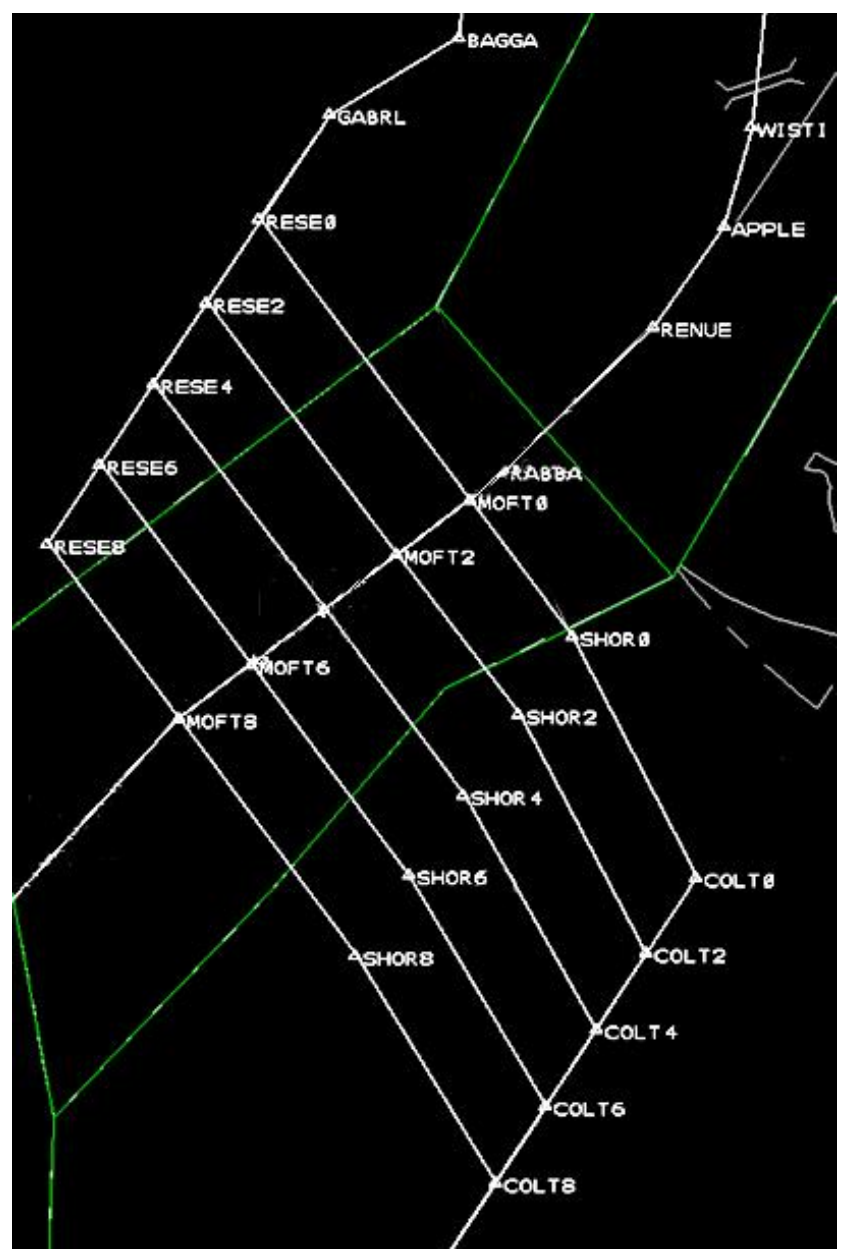

Figure 7. Preliminary Departure Route "Family"

\section{Aircraft's Trajectory and ETA Prediction}

For each of the pre-defined departure route options, there is a unique point of intersection with the arrival flow. The tool calculates the departure aircraft's ETA at the crossing point using the aircraft's current trajectory and speed [10]. The RCT then computes the anticipated location of the arrival aircraft at the time of the departure aircraft's crossing-point ETA. The resulting straight-line distance between the predicted location of the arrival and the crossing point is then displayed to the controller as the predicted separation value.

\section{Graphical User Interface (GUI) of RCT}

The development of the user interface was focused on providing relevant information to controllers. Two types of information were displayed: text information in a table form, and relative distance was graphically displayed with lines on the radar scope.

The RCT makes use of ETA calculations to generate predicted relative locations of the departure and arrival aircraft with respect to the path intersection point. By using the ETA to this shared point, numerous parameters can be computed.

\section{Display of Table of Values}

The RCT displays a table of values with route options and corresponding separation values for a departure aircraft. The table shown in Figure 8 consists of five columns of information. The first column displays the departure route name, or SID. The second and fourth columns, respectively, contain the arrival aircraft that will cross the intersection point directly before and after the departure aircraft. For clarity, these arrival aircraft are referred to as the leading and trailing arrivals. The third and fifth columns then, represent straight-line distances between the departure and leading arrival and the departure and trailing arrival-these two distances are computed using the predicted locations of the aircraft at the time the RCT predicts that the departure will cross the intersection point. 


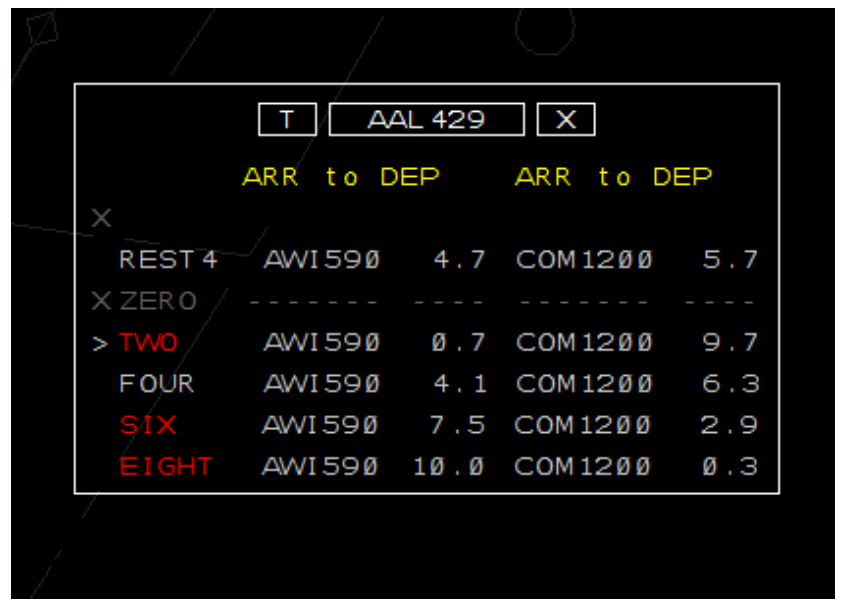

Figure 8. RCT Table of Values

Initial feedback from SMEs informed the decisions to avoid making the table a permanent menu. Instead, the table appeared on the perimeter of the radar display only when the controller clicked on the name of the currently-assigned SID in the departure aircraft's data block.

Changes in configuration for the RCT are implemented in MACS setup panels; these menus in MACS software allow researchers to define the parameters of the simulation. The display of the RCT table can be customized to show, for example, only the route names and predicted separation values, without the arrival aircraft call signs. The carrot symbol to the left of route TWO indicates the currently-assigned route. The grey text, dashes, and " $X$ " associated with the ZERO route indicate that ZERO is no longer a route option because the aircraft has already passed that SID's turn point.

When a controller clicks on the displayed name of the currently-assigned SID in that aircraft's datablock, then the straight-line distance between the predicted location of the arrival and the crossing point is displayed to the controller as the predicted separation value.

The separation values chosen were based on the path angles and the desired separation rules described earlier. The trailing arrival had to be at least $5.6 \mathrm{~nm}$ away $\left(S_{c}\right.$ in Figure 6A) from the departure when the departure was at the crossing point. The closest point of approach $\left(S_{\min }\right.$ in Figure 6A) then was $4 \mathrm{~nm}$. The $S_{c}$ value for the leading arrival was set to $4 \mathrm{~nm}$, with the closest point of approach $\left(S_{\min }\right.$ in Figure $\left.6 \mathrm{~B}\right)$ being $2.8 \mathrm{~nm}$. This assumes that the controller would issue a clearance to climb only after the aircraft routes diverged.

In Figure 8 several route names in the menu shown appear in red. This feature was added to indicate that if the controller chose that SID, a minimum separation between the departure and either the leading or trailing arrival was expected to be violated. MACS configuration options for the RCT evolved along with the RCT capabilities. The setup panel was expanded to include a field to define the minimum straight-line separation distance between departure and leading or trailing arrival and for determining when to display table values in red. A later iteration of the RCT's setup panel included the ability to define separate values for the minimum acceptable straight-line distance between the departure and leading arrival and between the departure and trailing arrival.

With the help of SMEs' feedback, researchers discovered multiple ways of redefining separation values to provide controllers with more relevant information. For example, SMEs confirmed that, per divergence rules, the distance between the leading arrival and the departure aircraft after the leading arrival has crossed the intersection point was not as critical to the controller's decision about climbing the departure. Therefore the segment containing the separation between the departure and leading arrival was modified to be computed based on the leading arrival aircraft's ETA at the intersection rather than using the departure aircraft's ETA at the intersection. This reduced clutter by showing only the relevant information. Once the leading aircraft crossed the intersection, its data could be removed from the display. The RCT setup panel in MACS was thus updated so researchers could choose whether to use the ETA of the departure or of the leading arrival at the intersection to calculate the predicted separation between these two aircraft.

\section{Graphical Display of Predicted Separation}

The tool's graphical display provides a visual representation of the values shown in the table and can be enabled in the MACS setup panel. Figure 9 shows the display after the controller has clicked on the SID in the data block of the departure aircraft COM1158 (towards the top of the scope). The table in the upper portion of Figure 9 appears on the perimeter of the radar scope after the SID has been clicked. The display shown in the lower portion of 
Figure 9 reflects the values of the currently-assigned route, denoted by a carrot on that line in the table. In this particular example, the departure is on the nominal REST4 (restricted route 4), a.k.a. SAFE route, and is predicted to be 3.5 nautical miles behind AWI535 and 10.8 nautical miles ahead of AWI275. Therefore, the graphical display depicts the respective predicted locations of AWI535 (leading arrival) and AWI275 (trailing arrival) at the time the departure should reach the intersection point. For visibility, two solid line segments are drawn to represent these predicted separations. The lines and the arrival aircraft locations (represented by the yellow and red circles at the ends of the lines shown in Figure 9) are drawn with the same color scheme shown in the table of values. The 3.5 nautical mile segment to the leading arrival's predicted position is therefore drawn in red, to indicate the value is below the threshold (set at 4 nautical miles). Three concentric circles are drawn at the point of intersection, and the currently-assigned departure route is also highlighted in the graphical display on the radar scope.

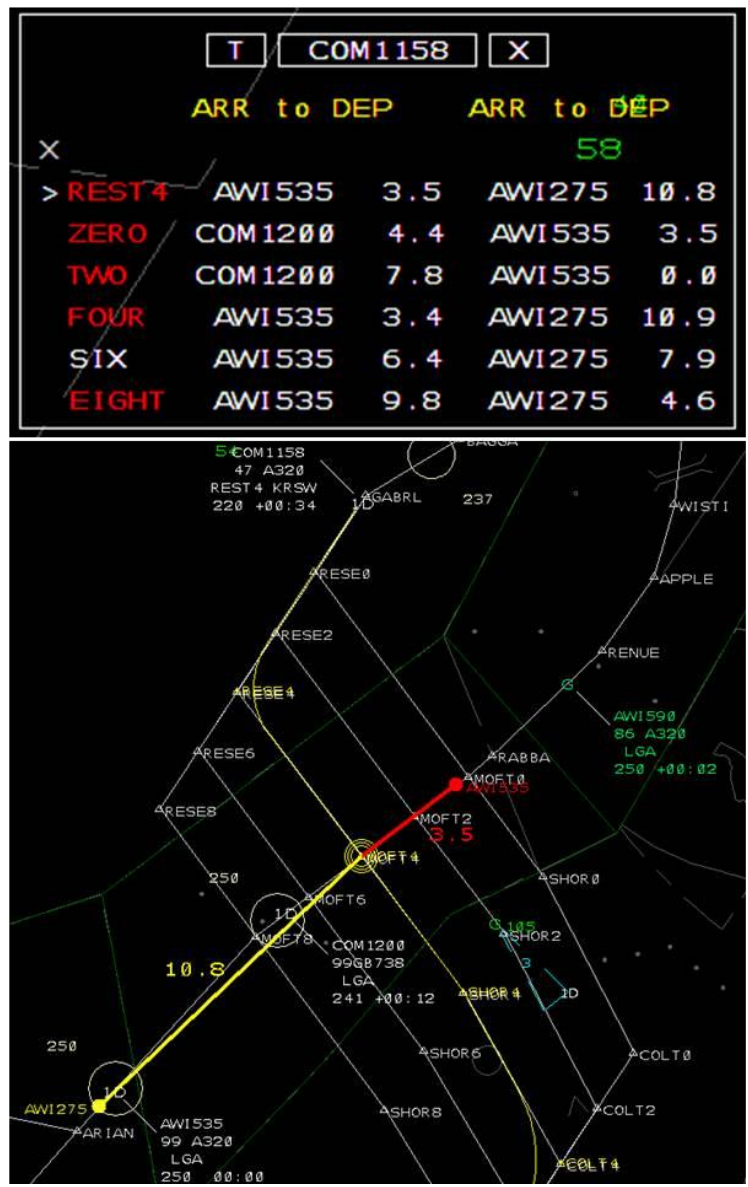

\section{Figure 9. SAFE Route Display}

Figure 9 above shows the display when the controller first clicked on the SID in the departure aircraft datablock. Figure 10 below shows the radar scope seconds later, when the controller is assessing different route options. As seen from the upper portion of Figure 10, the controller hovers over a route name in the table of values (in this case, route SIX). The graphical display on the scope updates as the controller hovers over different routes in the table of values. The same separation values shown in the table of values are displayed on the scope, with a yellow line between the departure and both the trailing and the leading arrival. The departure route that the controller is assessing is also highlighted, and the related intersection point is emphasized with concentric circles. In this example, the leading arrival, AWI535 is predicted to be 6.2 nautical miles ahead of the departure at the time of intersection, while the trailing arrival, AWI275, is predicted to be 8.1 nautical miles behind. Since both of these values are acceptable (and thus shown in yellow rather than red), the controller selects this route and updates the ground-side by clicking on the route name in the table. By choosing to instruct the departure to fly route SIX (and issuing this command to the pilot by voice), the controller has improved the likelihood of being able to climb the departure before the aircraft reaches the intersection point with the arrival flow. 

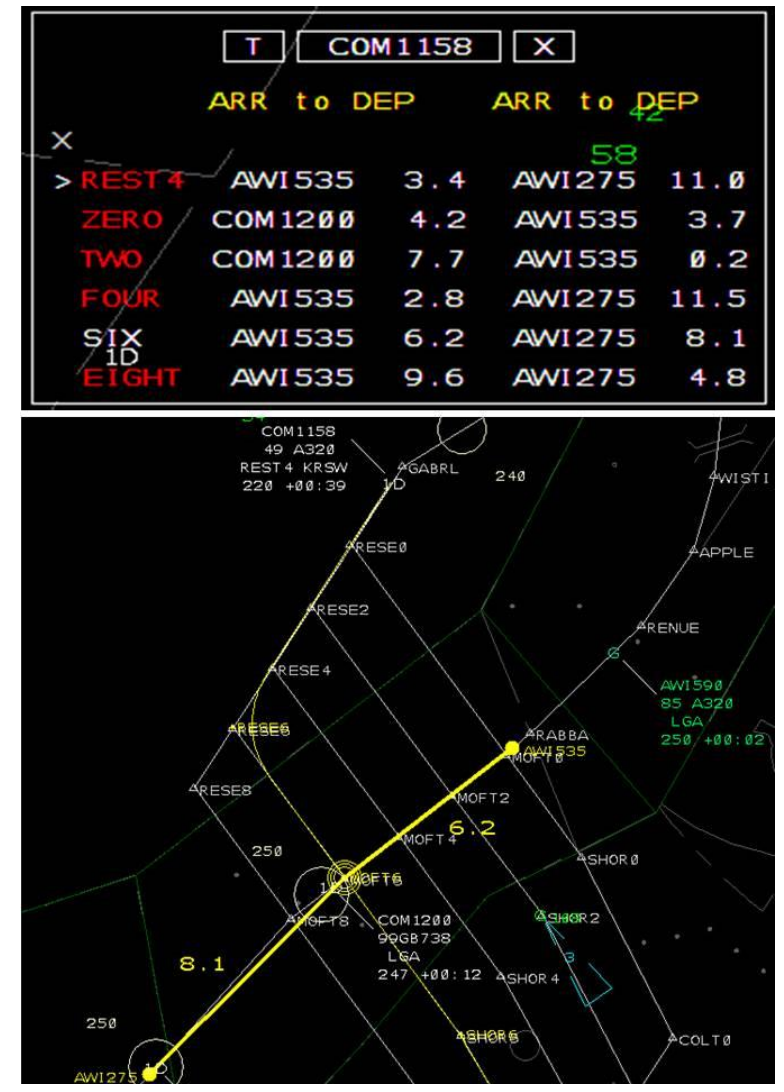

Figure 10. Selection of Route SIX

\section{Alternative Graphical Display of Predicted Separation}

An alternative display of the Table of Values is shown in Figure 11. In this display, the distance under the blue header "DEP to ARR," shows the position on the departure route where the departure is predicted to be when the lead arrival is at the crossing fix. On the radar scope, the line is blue and is depicted from the crossing fix onte the departure route. The top of the blue line indicates where the departure is predicted to be when the arrival is at the crossing fix, which is when route divergence will take place.
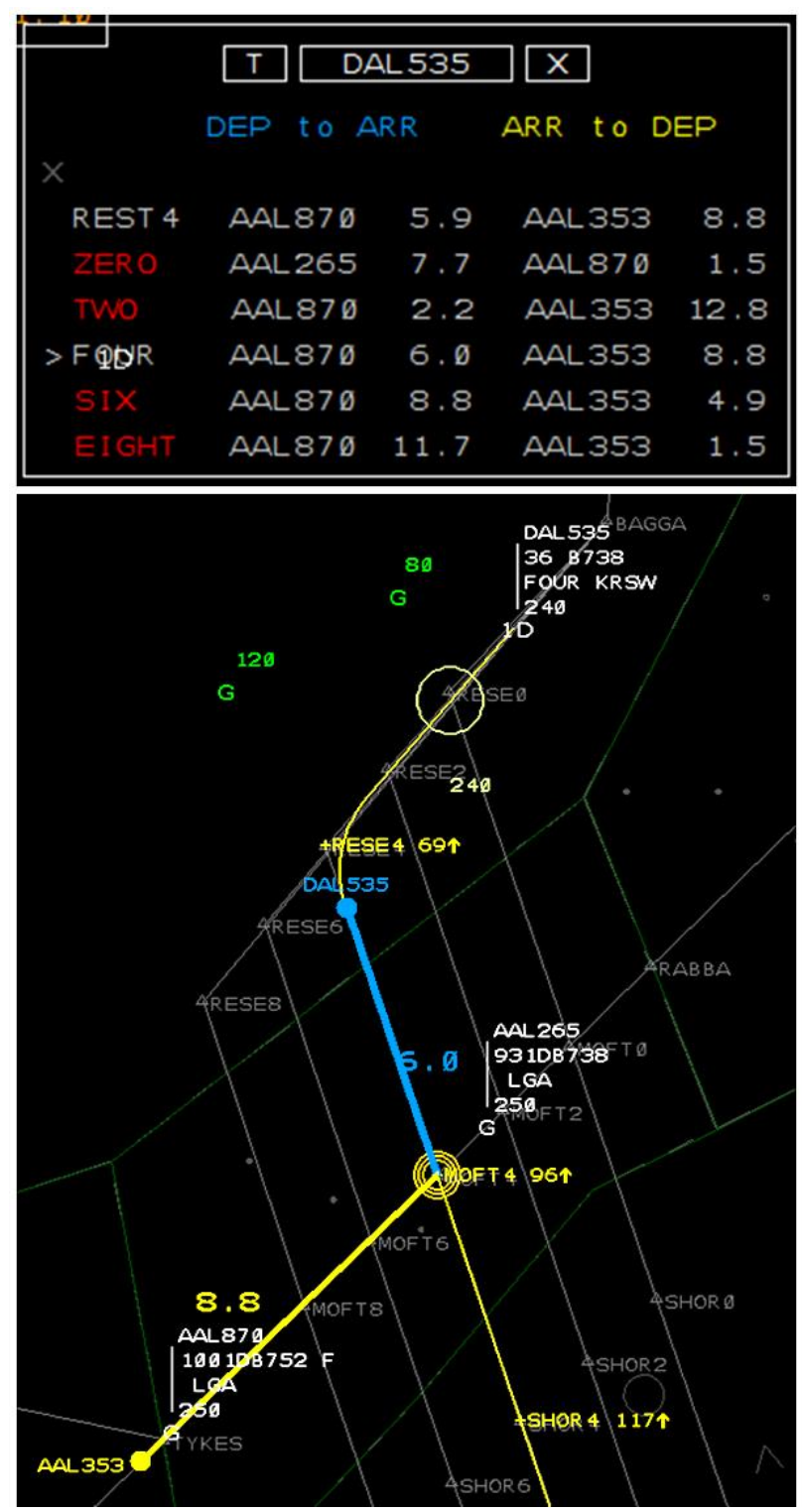

Figure 11. Separation for Route FOUR

When the value is less than the required minimum threshold plus a $1 \mathrm{~nm}$ buffer (here $4 \mathrm{~nm}$ ), the route name and the separation line turn red. Figure 12 shows one example with route TWO turning red. In this case the departure is estimated to be at $2.5 \mathrm{~nm}$ from the crossing fix before the aircraft are on diverging routes. This alternative graphical display was built, but not tested. 

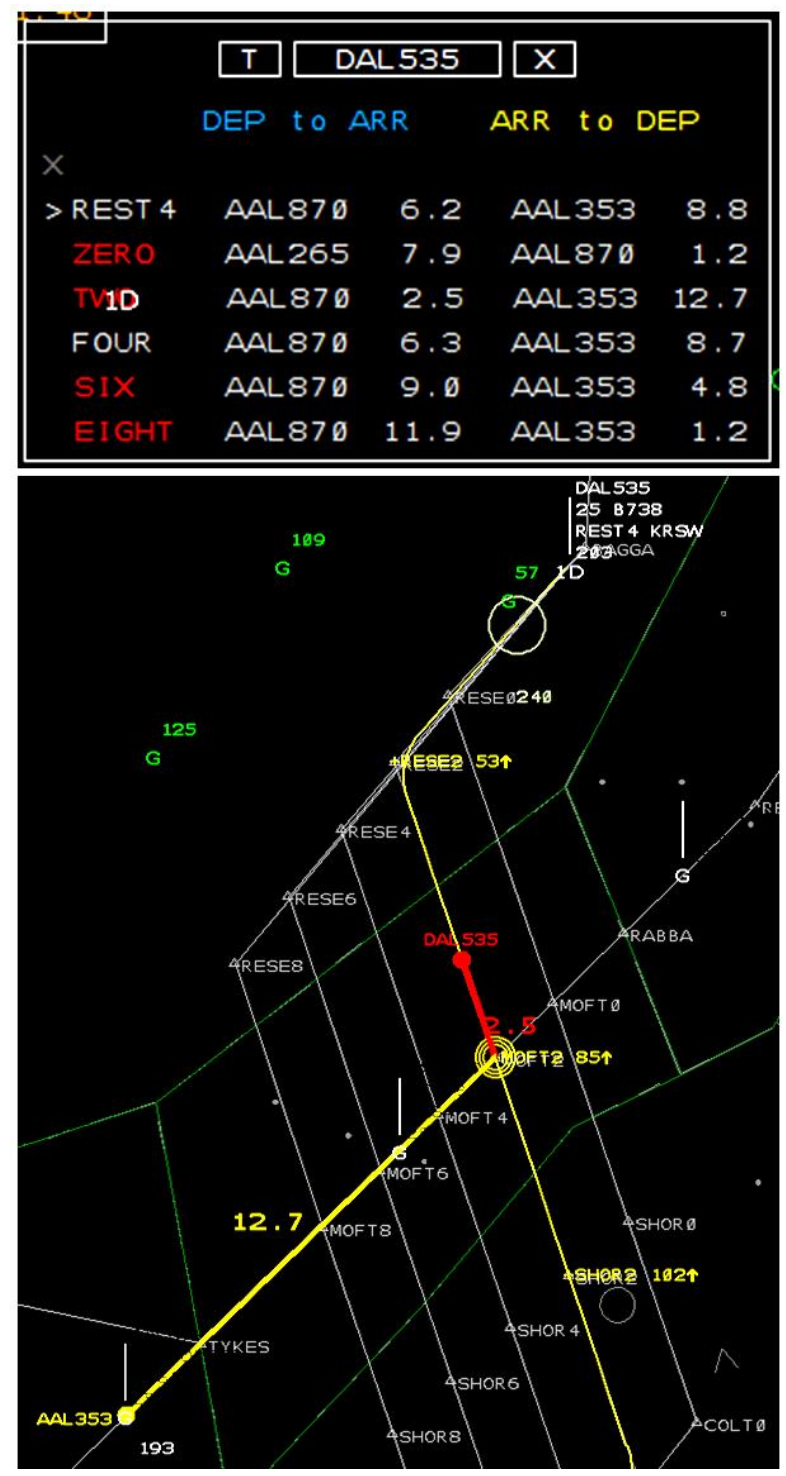

Figure 12. Separation for Route TWO

\section{Timeline}

A third component of the RCT is a timeline with times for both the arrival aircraft and the crossing departure aircraft. The shared arrival/departure timeline shown in Figure 13 reflects the same traffic situation depicted in Figures 9 and 10. The departure in question, COM1158, is shown in a different color than the arrivals and is placed between its leading and trailing arrivals. The timeline indicates that on route SIX, COM1158 will arrive at the crossing-point about 80 seconds behind lead arrival AWI535 and about 100 seconds ahead of trail arrival AWI275. Based on the displayed separations (6.2 and 8.1) at the crossing point the controller could climb COM1158 above $8,000 \mathrm{ft}$ well before it crossed the arrival flow at MOFT6 with the expectation of always having more than minimum lateral separation. The common arrival/departure timeline shown in Figure 11 offers similar information as the table and the graphical display, but on the basis of time rather than distance.

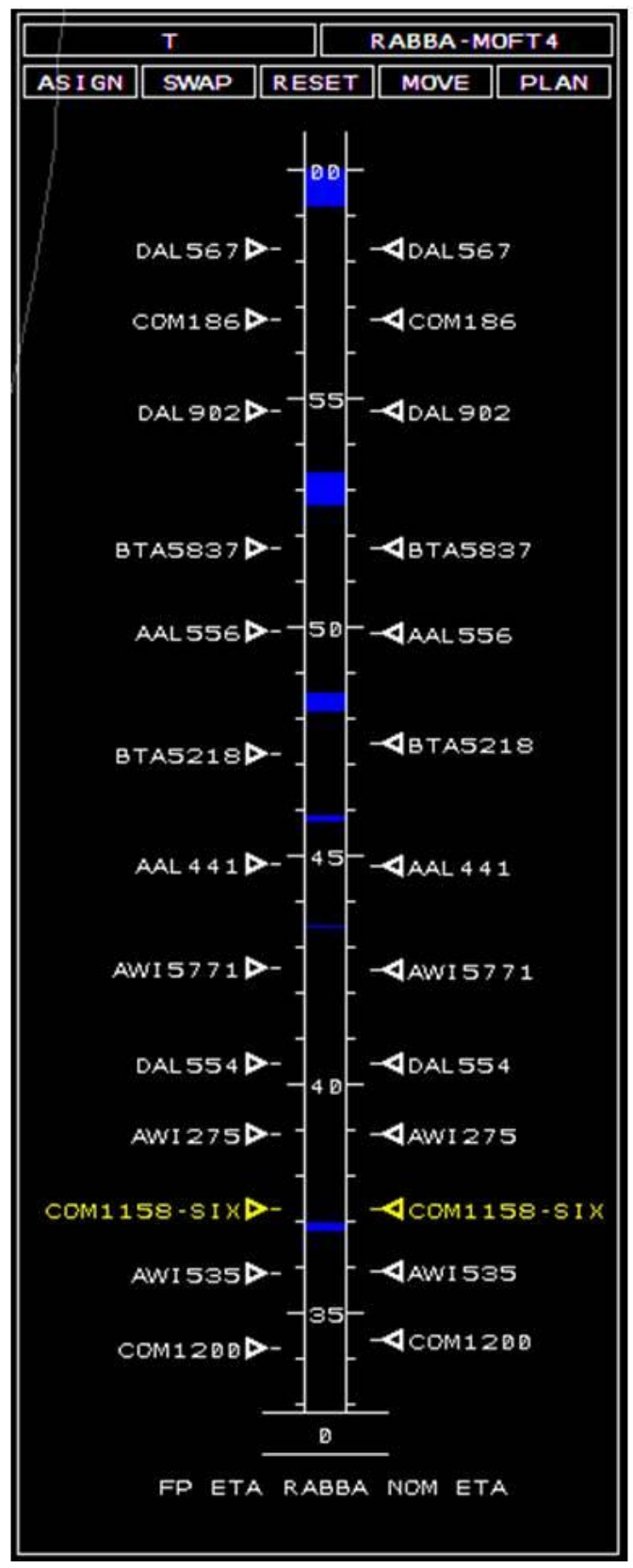

Figure 13. Shared Arrival/Departure Timeline

\section{Route Crossing Task Procedures}

The following procedures outline a possible use of the RCT in New York TRACON operations. Consider the route crossing task of climbing EWR 
departures through a LGA arrival flow; this is the responsibility of the controller working departure and arrival aircraft in the EMPYR sector in the New York TRACON. EWR Tower has scheduled departures into "gaps" in the LGA arrival flow; however, it is anticipated that the departures will takeoff either early or late. Seen in Figure 13, the gaps are shown as blue stripes in the center of the timeline. The blue gaps represent periods of time during which there is a sufficient time between consecutive arrival aircraft for a departure. Though these gaps, the EWR tower can clear an aircraft for takeoff.

On initial contact with the departure aircraft, the EMPYR controller climbs the departure to a safe altitude on the nominal route (a procedurally separated route under the arrival flow). The EMPYR controller then clicks on the SID of the departure aircraft's datablock, displaying the RCT in order to assess the other routes in the departure route family. The controller then decides on a lateral route based on the predicted relative distances given by the RCT. While controllers may adopt different strategies for which route to choose, the objective is to choose a route that minimizes the need to cap the departure's climb due to the arrival. In the event that all route options are red (i.e., predicted to have insufficient spacing between the departure aircraft and the neighboring arrivals), a route may still be selected because it offers the best chance of climbing the departure, even if that cannot be done immediately. A safe route is always available, as the departure just needs to be held at an altitude under the arrivals, on any of the lateral route options.

By hovering over the route name in the table, the RCT's graphical display information is shown, allowing the EMPYR controller to quickly evaluate which routes appear viable (similar to the depiction in Figure 9). By clicking on the route name in the RCT table, the EMPYR controller makes a route amendment, and must instruct the pilot to fly the new route. If the controller stays with the nominal route, no amendment is necessary. If the RCT gives a steady, acceptable predicted separation between the departure and the trailing arrival, the controller may clear the departure to climb after the lead arrival aircraft has passed the intersection point (leveraging the divergence rules). The transfer of communications to the next sector can take place after the departure clears the arrival flow.
The RCT is useful not only because it helps to predict separation, but also because it allows the controller to update the filed route in the ground system. It can also be used to guide the controller in vectoring aircraft if the pilots in the flight deck are unable to load a new route.

\section{Design Iterations: Explored Solutions Departure Meter Fix Scheduling}

An early version of the RCT included the delay times at a downstream meter fix in the table of values. One complication with designing the RCT involved understanding whether or not the route crossing task and the meter fix delivery tasks could be treated separately. For example, a route that provided the best possibility for climbing the departure aircraft before reaching the arrival flow might be chosen, but that route could alter the ETA at the meter fix. The reason for this discrepancy is a difference in flight time across routes: every route except for the SAFE route (a route capped below the LGA arrivals that was used as the nominal route) lacks an altitude restriction at the intersection point; the SAFE route does have an altitude restriction at that point. Referring back to the route geometry discussion, the departure route family may be packaged together in the shape of a parallelogram so that the flying time to a downstream meter fix is not affected, but there is a difference between the SAFE route and any other route because of the increased speed with which the planes may travel when climbing above $10,000 \mathrm{ft}$. Therefore, as a proof of concept, the route crossing task was treated separately from the meter fix task in the 2014 HITL. This meant that the EMPYR controller's sole goal was to climb the departures when practicable, regardless of schedule at the meter fix. For this reason, the column of delay times at the meter fix in the RCT table was not displayed.

\section{Safe Route Questions}

Another question in the design process addressed the SAFE route and whether it should ever turn red in the table and on the graphical display. Although the lateral separation could certainly be less than the required minimum on the SAFE route, the departures were always separated from the arrivals by altitude on this route. To maintain consistency in terms of what the RCT values represented, the SAFE route would turn red when the predicted minimum 
lateral separation at the crossing point was less than the specified minimum. However, to turn a SAFE route red seemed contradictory. One proposal was to put the SAFE route on the top of the list and to not show any values associated with the route, but just to have it as an option to return to on the route list. A complication, however, is that any route can be "safe," regardless of its lateral positioning by restricting the departure's altitude along the route. Further user-interface design work is needed to address these concerns.

\section{Stability of Tool Updates}

One major improvement of the RCT over other previously studied passive tools is the continuous prediction updates, which refresh the ETA data every radar-hit. A problem with this dynamic characteristic, however, is that the RCT's predictions can fluctuate. A route name displayed in the table, for example, can rapidly change from red to white and vice-versa. This led to a discovery which improved the RCT's usability: the controller using the tool should wait a few seconds for the values given by the RCT to stabilize because the initial values were necessarily "jumping" with the imprecise ETA data at the beginning of the aircraft's climb.

\section{Ghost Position}

Although the need for the tool to be dynamic was clear from previous simulations, an appropriate way of presenting this constantly changing information remained less clear. Alternative solutions to presenting information to aid controllers in the route crossing task were debated; one suggestion was to have a "ghost" aircraft. A ghost aircraft would indicate a hypothetical location of the departure aircraft as if it were on the same path as the arrivals. An example of this is shown in Figure 12, where the orange dot that is the ghost marker moves along the purple arrival trajectory so as to arrive at the route crossing point at the same time as the departure. One drawback to this way of presenting information, however, is that the ghost marker can be off the scope for the routes that have longer path lengths. It was therefore decided that for the June HITL, the ghost marker would not be used and instead, the combination of graphical RCT display, table of values, and shared arrival/departure timeline shown in Figures 8 through 11 would be used.

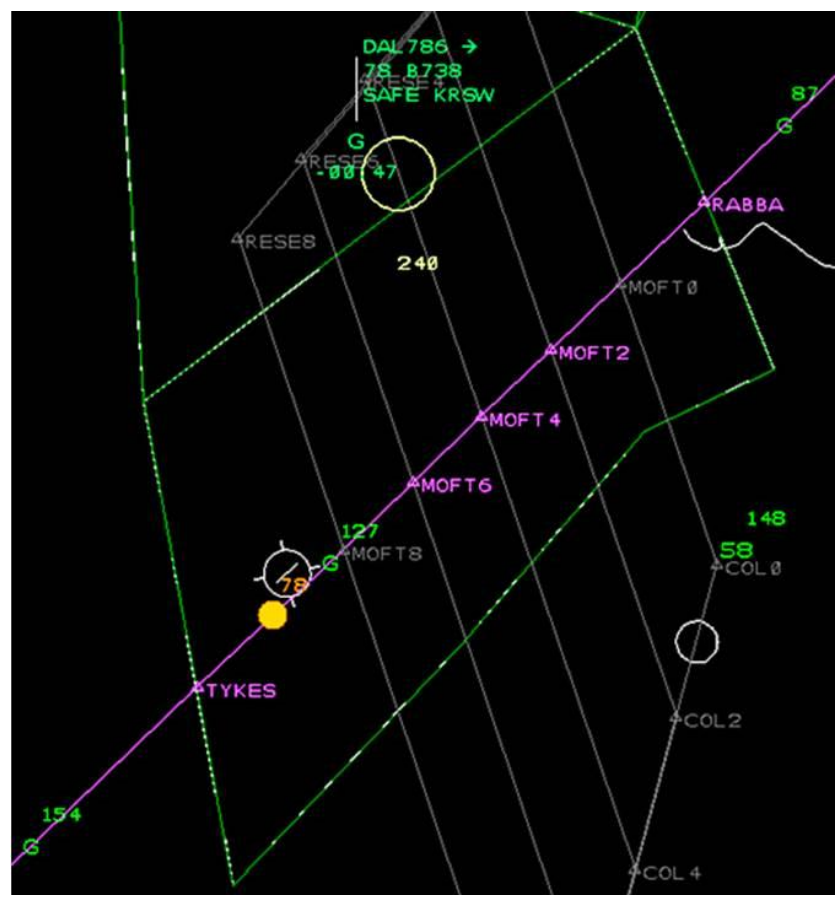

Figure 12. Ghost Marker

\section{Other Interface Design Considerations}

There are a few other interface design questions that need to be considered. For example, the red warning lines on the graphical RCT appear when there are small separation values and therefore the lines themselves are small and hard to see, while they are the most important. Further, in an effort to minimize clutter on the controllers' scopes, separation distances of greater than a configurable distance, e.g. $20 \mathrm{~nm}$, are not displayed. This nondisplay of arriving aircraft, however, may unintentionally indicate to controllers that there are no arrivals present. These considerations, along with further analysis of the simulation results and feedback from the controllers will inform the next steps in tool design.

\section{Preliminary Results and Observations}

Preliminary results from the June HITL indicate that controllers were able to climb more departures when using the RCT tool compared to a baseline condition without the tool. Controller feedback was positive, with one controller stating that "The crossing tool was very accurate! Better than my eyes when the aircraft were far apart." Researchers' observations and controller responses to post-sim 
surveys indicate that controllers developed their own rules of thumb for interacting with and making use of the RCT. For example, some controllers were observed to select the departure route that gave the smallest separation between the departure and leading arrival aircraft (without being red); they would then climb the departures after the leading arrival cleared the intersection point, as long as the separation to the trailing arrival was maintained. Other controllers would notice fluctuating separation values being given by the RCT, and would therefore adjust speed to "lock in" the viable, non-red routes. For example, in borderline cases where the separation values are just 0.1 nautical mile above the threshold for turning red, a controller may increase (or reduce, depending on the situation) the departure aircraft's speed in order to increase the separation value so that the value is less likely to dip below the threshold as the value is regularly updated.

During tool development, it was suggested that controllers be unable to make route amendments if they were red. However, controllers often selected a red route in anticipation of the leading arrival passing the intersection point causing the route to become a viable climbing option again, due to divergence. In rare instances where all route options were red, the controllers showed a tendency to select the routes which gave greater separation values between the departure and trailing arrival, rather than between the departure and the leading arrival.

In the no-tools (baseline) condition of the study, controllers reported that in certain cases, it was difficult to tell if the minimum separation between a departure and arrival would be maintained. During this baseline condition, one strategy adopted by controllers was to use J-Rings to assess separation with arrivals. In today's operations, controllers can choose to display a J-Ring around particular aircraft; the J-Ring encircles a target on the radar scope and has a radius defined by the controller. In the postsimulation questionnaire of this study, a controller reported using $3 \mathrm{~nm}$ and $5.6 \mathrm{~nm}$ radius J-Rings on the leading and trailing arrival aircraft respectively, to assess the separation between the departure and these two relevant arrivals. The choices of 3 and $5.6 \mathrm{~nm}$ for J-Ring radius sizes were similar to the previously discussed numerical values used to define thresholds in the MACS setup panels for the RCT. This J-Ring strategy not only demonstrated that the controller was comfortable with the thresholds used for the RCT, but that the design of the RCT inspired the controller to create a simpler version of it.

While the workload for the person managing both arrivals and departures was generally acceptable in this study, the controller's one task was essentially to climb departures when possible. Controllers suggested that workload would increase if the arrival flow were less well-conditioned than the flow presented to the controllers in the June simulation. An interesting investigation might include an arrival flow with aircraft not quite on schedule, thereby adding the task of conditioning the arrivals to the controller's responsibility. A further look into the workload, not only in terms of cognitive demand but also the time required for the route crossing task, would give even more insight into the requirements for a route crossing task aid.

\section{Concluding Remarks}

To summarize, previous research had suggested a need for a dynamic decision-support tool to aid controllers in the task of safely managing intersecting departure and arrival flows in a shared airspace environment. Iterative development with controllers uncovered many interesting aspects regarding the feasibility and acceptability of such a route crossing tool. Preliminary results from a HITL indicated that the RCT tool enabled more aircraft to be climbed than were climbed in a no-tool condition; controller feedback was also positive. An especially interesting observation was that the controllers who worked with this tool made use of different aspects to varying degrees, and multiple ways of using the tool emerged, even though the underlying tool structure was the same. Because of the wide set of issues explored, this research is an example of the value of including subject matter experts early on in an iterative and flexible development process of NextGen decision-support tools.

\section{References}

[1] Clarke, John-Paul, Liling Ren, Evan McClain, David Schleicher, Sebastian Timar, Aditya Saraf, Donald Crisp, Richard Gutterud, Ryan Laroza, Terence Thompson, Carolyn Cross, Taryn Lewis, and Michael Madson, 2010, Evaluating concepts for metroplex operations, Proceedings of the Aviation 
Technology, Integration, and Operations Conference, Fort Worth, Texas, AIAA.

[2] Chevalley, Eric, Bonny Parke, Paul Lee, Faisal Omar, Hyo-sang Lee, Nancy Bienert, Joshua Kraut and Everett Palmer, 2013, Scheduling and separating departures crossing arrival flows in shared airspace, Proceedings of the 32nd Digital Avionics Systems Conference, Syracuse, New York, IEEE.

[3] Chevalley, Eric, Paul Lee, Bonny Parke, Faisal Omar, Nancy Bienert, Joshua Kraut, and Everett Palmer, 2014, Decision support tools for climbing departure aircraft through arrival airspace, $33^{\text {rd }}$ Digital Avionics System Conference, Colorado Springs, Colorado, IEEE.

[4] Parke, Bonny, Eric Chevalley, Paul Lee, Faisal Omar, Joshua M. Kraut, Kari Gonter, Abhay Borade, Conrad Gabriel, Nancy Bienert, Cindy Lin, HyoSang Yoo, and Everett Palmer, 2014, Coordination between sectors in shared airspace operations, $33^{\text {rd }}$ Digital Avionics System Conference, Colorado Springs, Colorado, IEEE.

[5] Capozzi, Brian, Stephen Atkins, and Seongim Choi, 2009, Towards optimal routing and scheduling of metroplex operations, Proceedings of the Aviation Technology, Integration, and Operations Conference, Hilton Head, South Carolina, AIAA.

[6] Xue, Min, Shannon Zelinski, 2012, Optimal integration of departures and arrivals in terminal airspace, Proceedings of the Guidance, Navigation, and Control Conference, Minneapolis, Minnesota, AIAA.

[7] Jung, Yoon, Douglas Isaacson, 2002, Design concept and development plan of the Expedite Departure Path (EDP), Proceedings of the Aviation Technology, Integration, and Operations (ATIO) Conference, Los Angeles, California, AIAA.

[8] Jung, Yoon, Douglas Isaacson, 2003, Development of conflict-free, unrestricted climbs for a terminal area departure tool, Proceedings of the Aviation Technology, Integration and Operations Conference, Denver, Colorado, AIAA.

[9] Wang, Jianzhong, Paul Chang, and Koushik Datta, 2003, Life-Cycle cost/benefit assessment of Expedite Departure Path (EDP), Prepared for NASA Ames Research Center, Contract No. NAS2-98074.

[10] Prevot, Thomas, Nancy Smith, and Everett Palmer, 2006, The Airspace Operations Laboratory
(AOL) at NASA Ames Research Center, Proceedings of the Modeling and Simulation Technologies Conference, Keystone, Colorado, AIAA.

[11] Globus, Al, Richard Jacoby, Thomas Prevot, and James Wong, 2011, Rapid generation and utilization of four-dimensional trajectories for air traffic control and management applications in MACS, Proceedings of the Infotech@Aerospace Conference, St. Louis, Missouri, AIAA.

[12] Federal Aviation Administration, 2014, Order JO 7110.65V, Chapter 5, Section 5, Part 7, Washington, D.C., U.S. Department of Transportation. Available online at http://www.faa.gov/documentLibrary/media/Order/A TC.pdf

\section{Acknowledgements}

The authors are grateful for the contributions made by the Sharing of Airspace Resources (SOAR) research team, as well as those of the other researchers and developers of the Airspace Operations Laboratory. Additionally, credit is owed to the insights provided by the Subject Matter Experts who informed this work as well as the many controller and pilot participants.

33rd Digital Avionics Systems Conference October 5-9, 2014 